\title{
Generation and Annihilation of Magnetic Helicity in Active Regions
}

\author{
K. Kusano \\ Graduate School of Advanced Sciences of Matter, Hiroshima University, \\ Higashi-Hiroshima, Hiroshima 739-8530, Japan
}

\begin{abstract}
Generation and annihilation processes of magnetic helicity in solar coronal active regions are investigated based on the observations and the simulations. We first examined the reliability of the numerical techniques, which enable to measure the magnetic helicity flux through the photosphere based on the magnetogram data. Secondly, in terms of the new technique, we found that magnetic helicities of the both signs are simultaneously injected into active regions. Motivated by this result, finally, we investigated the nonlinear process of the magnetic helicity annihilation, using the three-dimensional numerical simulations. The simulations clearly indicated that the helicity reversal can cause the eruption of large-scale plasmoid through the nonlinear process of the resistive instability growing on the helicity inversion layer. From these studies, we point out that the annihilation of magnetic helicity is a key process for the onset mechanism of solar flares.
\end{abstract}

\section{Introduction}

Magnetic helicity is believed to be an important quantity to understand the solar coronal activities, such as flares and the coronal mass ejections (CMEs). However, the previous studies of magnetic helicity in the solar corona were restricted mainly to the theoretical and conceptual models. One reason of that is attributed to the fact that the measurable magnetic field in the solar corona is limited only to the photospheric and coromospheric levels, while the helicity is defined as the spatial integration over the whole domain.

Recently, new methodologies to evaluate the helicity flux out of the sun have been proposed (Kusano et al. 2002, Démoulin and Berger 2003). In this paper, first we will review the principle of the helicity measurement and examine the reliability using the benchmark model. Secondly, we will discuss about the possibility that the annihilation of magnetic helicity works as the trigger mechanism of solar flares, based on the measurement of the helicity flux and the three-dimensional numerical simulations.

\section{Measurement of Helicity Flux}

According to Berger and Field (1984), the helicity flux across a plane $S$ is described by

$$
\dot{H}=2 \int_{S} \boldsymbol{A}_{P} \times \boldsymbol{E} \cdot \boldsymbol{n d S}
$$



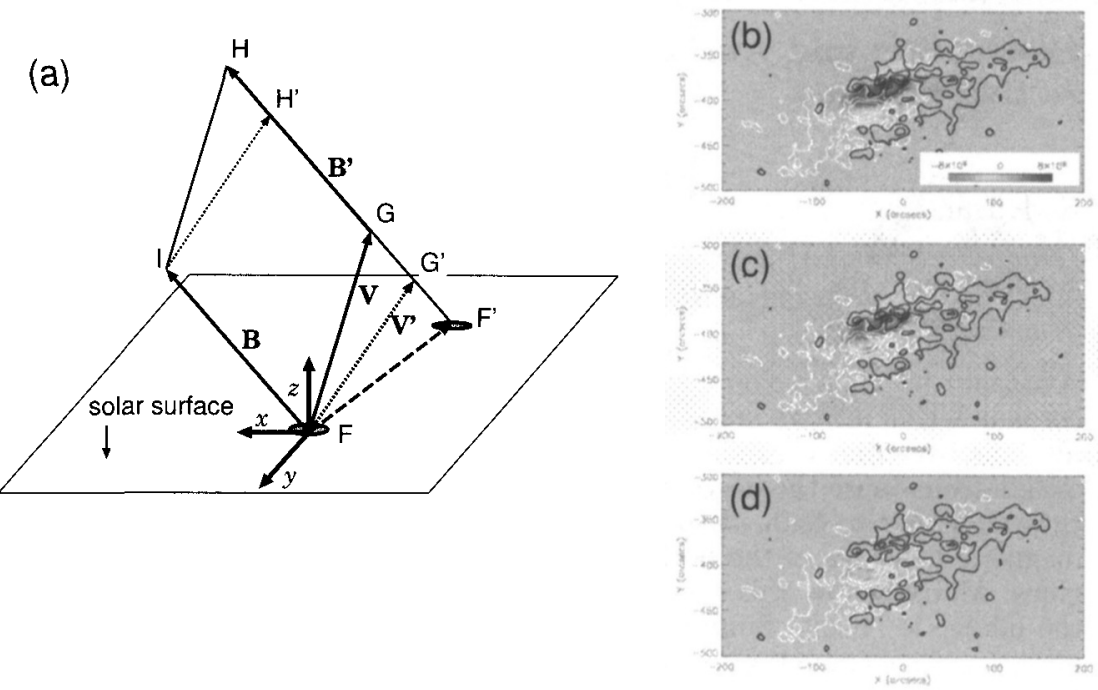

Figure 1. (a) The illustration of field line variation on the solar surface. (b) to (d) The benchmark test of the helicity flux measurement. The color shading and the contours indicate the helicity flow and the magnetic field, respectively. (b) is the correct answer. (c) is the result by the combination method of the induction equation and the LCT. (d) is the result by the LCT method.

where $\boldsymbol{A}_{P}$ is the vector potential of the current free field, which is derived from the magnetic field normal to the boundary. It indicates that the electric field $E$ is a key quantity for the helicity measurement. In the ideal magnetohydrodynamic (MHD) regime, the electric field is related with the plasma velocity $\boldsymbol{V}$ through the generalized Ohm's law $\boldsymbol{E}=-\boldsymbol{V} \times \boldsymbol{B}$.

In Fig.1a, let us consider the situation that the magnetic field line $\boldsymbol{B}$ is transfered by the velocity $\boldsymbol{V}$ for a unit time into $\boldsymbol{B}^{\prime}$. In the case, the electric field vector $\boldsymbol{E}$ corresponds to the parallelogram FGHI, in which the amplitude and the direction are indicated by the area and the normal vector, respectively. Here, we should note also that the parallelogram made by any vector connecting the two field lines, for instance FG'H'I, has the same area and the same normal direction as FGHI. It means that any vector connecting the two field lines forms a consistent virtual velocity $\boldsymbol{V}^{\prime}$, which satisfies $\boldsymbol{E}=-\boldsymbol{V}^{\prime} \times \boldsymbol{B}$. Thus, the helicity flux is able to be derived without the measurement of the real velocity $\boldsymbol{V}$, if we can detect the virtual velocity $\boldsymbol{V}^{\prime}$.

Recently, two different ways were proposed for the derivation of the virtual velocity. Démoulin \& Berger (2003) pointed out that, since the vector along the foot point motion $\mathrm{FF}^{\prime}$ forms the virtual velocity, the apparent velocity derived by the local correlation tracking (LCT) of the magnetograms could be used for the helicity measurement. This proposition is valid, if and only if each foot point of field line is identified. However, there is no guarantee that the apparent motion derived by the LCT technique satisfies the definition of the virtual velocity, 
because we can observe only the magnetic pattern on the photospheric surface rather than each foot point of the field lines. Furthermore, when the field line is tangential to the solar surface, the measurement of the foot point motion could be much difficult, because the apparent velocity must diverges into infinity,

On the other hand, Kusano et al. (2002) demonstrated that the virtual velocity can be derived by inversely solving the induction equation of the normal component on the solar surface,

$$
\partial \boldsymbol{B}_{n} / \partial t=\left[\nabla \times\left(\boldsymbol{V}^{\prime} \times \boldsymbol{B}\right)\right]_{n},
$$

in which the magnetic field vector $\boldsymbol{B}$ and the variation of the normal component $\partial \boldsymbol{B}_{n} / \partial t$ are given by the magnetograph observation, and only $\boldsymbol{V}^{\prime}$ remains as an unknown. This method has an advantage that the solution automatically satisfies the definition of the virtual velocity.

However, the induction equation cannot determine the velocity component orthogonal both to the vertical vector $\boldsymbol{B}_{n}$ and the tangential vector $\boldsymbol{B}_{t}$ of the magnetic field (i.e. the $y$ component in Fig.1a). Therefore, we need the help of the LCT technique in order to determine $V_{y}^{\prime}$. Here, notice that the $y$ component of the foot point motion does not diverge even if the field line is tangent to the solar surface. It is another advantageous point of this method.

Figures 1(b) to 1(d) represent the result of a benchmark test of the two methods. Here, we first calculated the three-dimensional linear force-free field, which satisfies $\nabla \times \boldsymbol{B}_{l f f}=\alpha \boldsymbol{B}_{l f f}$, from the SOHO/MDI data. Then, the helicity flow in the case that the whole active region is uniformly elevated was derived by the two different methods only from the fields on the two horizontal cross sections at different levels, i.e. $\boldsymbol{B}_{l f f}\left(z=z_{0}\right)$ and $\boldsymbol{B}_{l f f}\left(z=z_{0}+\Delta z\right)$. The result clearly indicates that the combination of the induction equation and the LCT technique can derive the better answer than the result only by the LCT.

Our measurement with this combination technique revealed that positive and negative magnetic helicities are simultaneously injected in flare productive active regions (Kusano et al. 2002). As a result, the structure of magnetic helicity in the active regions were much complicated both in time and space, so that even the sign of the magnetic helicity often changes (Kusano et al. 2003)

This new finding suggested another possibility of the mechanism of energy liberation. If positive and negative helicities coexist within an active region, magnetic reconnection could cancel the helicity by merging the counter helicity fluxes. If it does, the magnetic field may relax to the helicity-free field, and the free energy should be released. This process can be understood as the annihilation of magnetic helicity.

\section{Simulation of Helicity Annihilation}

In order to examine the hypothesis of the helicity annihilation, the nonlinear MHD process, in which the foot-point shear motion reverses the pre-loaded magnetic helicity, was investigated using the numerical simulations. As a result, it was clearly demonstrated that the reversal of magnetic helicity can cause a large scale eruption of the magnetic arcade through the following processes. First, the resistive tearing mode instability grows on the helicity inversion layer. Secondly, magnetic reconnection driven by the tearing mode annihilates the 


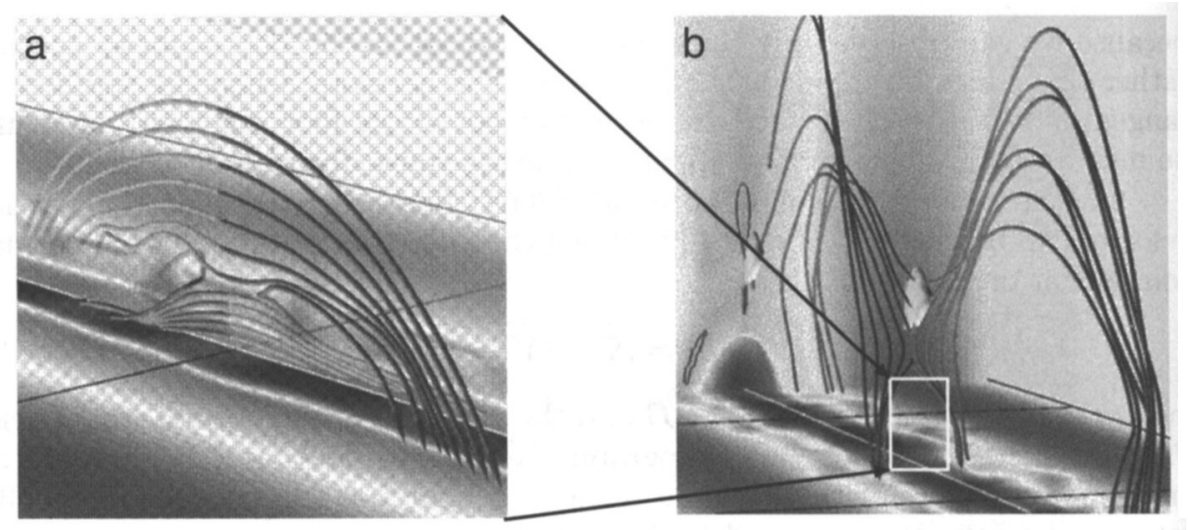

Figure 2. Magnetic field line structure in the numerical simulation of the helicity annihilation process.

positive and negative magnetic fluxes parallel to the magnetic neutral line, as indicated by red lines in Fig.2(a). Thirdly, the flux annihilation is followed by collapse of the magnetic arcade, so that the vertical current sheet is generated. Fourthly, the new current sheet drives the second reconnection, which causes the plasmoid ejection, as seen in Fig.2(b). Finally, the total system reaches a loss-of-equilibrium state, and the whole magnetic arcade is erupted upward.

This model can well explain the explosive property of flare onset, even though the growth rate of the original instability is much slower than the MHD time-scale. Although the reality of our model in the coronal plasma is still unclear, the annihilation of magnetic helicity is a promising concept for the onset mechanism of solar flares and the further investigation is required.

\section{References}

Berger, M.A., Field, G.B. 1984, J. Fluid Mech., 147, 133

Démoulin, P. \& Berger,M.A. 2003, Solar Phys., 215, 203

Kusano, K., Maeshiro, T., Yokoyama, T., \& Sakukrai T. 2002, ApJ, 577, 501

Kusano, K., Maeshiro, T., Yokoyama, T., \& Sakukrai T. 2003, Advances in Space Research, 32, 1917 